Research Paper

\title{
Increment of growth factors in mouse skin treated with non-thermal plasma
}

\author{
Byul Bo Ra Choi ${ }^{1,2, *}$, Jeong Hae Choi ${ }^{1,2,}$, , Jeong Ji², Ki Won Song 3 , Hae June Lee ${ }^{4}$ and Gyoo Cheon Kim² ${ }^{\circledR}$ \\ 1. Feagle Co., Ltd., Yangsan 50614, Republic of Korea \\ 2. Department of Oral Anatomy, School of Dentistry, Pusan National University, Yangsan 50612, Republic of Korea \\ 3. Department of Biochemistry, College of Life Science and Biotechnology, Yonsei University, Seoul 03722, Republic of Korea \\ 4. Department of Electrical Engineering, Pusan National University, Busan 46241, Republic of Korea \\ *These authors contributed equally to this work. \\ $\triangle$ Corresponding author: Gyoo Cheon Kim, Department of Oral Anatomy, School of Dentistry, Pusan National University, Yangsan 626-870, Republic of Korea. \\ Email: ki91000m@pusan.ac.kr; Tel: 82-51-510-8243; fax: 82-51-510-8241. \\ (C) Ivyspring International Publisher. This is an open access article distributed under the terms of the Creative Commons Attribution (CC BY-NC) license \\ (https://creativecommons.org/licenses/by-nc/4.0/). See http://ivyspring.com/terms for full terms and conditions.
}

Received: 2018.03.28; Accepted: 2018.06.30; Published: 2018.07.30

\begin{abstract}
Non-thermal plasma (NTP) has several beneficial effects, and can be applied as a novel instrument for skin treatment. Recently, many types of NTP have been developed for potential medical or clinical applications, but their direct effects on skin activation remain unclear. In this study, the effect of NTP on the alteration of mouse skin tissue was analyzed. After NTP treatment, there were no signs of tissue damage in mouse skin, whereas significant increases in epidermal thickness and dermal collagen density were detected. Furthermore, treatment with NTP increased the expression of various growth factors, including TGF- $\alpha$, TGF- $\beta$, VEGF, GM-CSF, and EGF, in skin tissue. Therefore, NTP treatment on skin induces the expression of growth factors without causing damage, a phenomenon that might be directly linked to epidermal expansion and dermal tissue remodeling.
\end{abstract}

Key words: Non-thermal plasma, Skin regeneration, Clinical application, Growth factor

\section{Introduction}

The skin is the largest organ of the body, accounting for approximately $16 \%$ of the total body weight of an adult [1]. Skin is constantly exposed to external environments and serves as a protective barrier [2], protecting the body against exogenous hazards, including biological infection, chemical substances, and UV [3]. The skin is composed of two layers, the epidermis (upper layer) and the underlying layer of dermis (lower layer). The epidermis is mainly composed of keratinocytes (approximately $90 \%$ of the epidermis) [4,5]. Although the dermis contains several functional tissues, including nerves, hair follicles, and sweat glands [6], it is mainly comprised of collagen, fibroblasts, and elastin fibers that provide nutrients [7].

Maintenance of healthy skin is important not only for anti-aging and rejuvenation, but also for wound healing. Skin aging accompanies reduction in collagen, decrease in various growth factors, and loss of fibroblasts [8-10]. To protect skin from aging, keratinocytes in the epidermis need to proliferate and fibroblasts in the dermis need to actively produce extracellular matrix proteins such as collagen and elastin fibers [7, 11]. For successful healing of skin wounds, a series of events should proceed favorably, including coagulation, inflammation, re-epithelialization, wound contraction, extracellular matrix rearrangement, and angiogenesis [12-14]. The skin beauty market has been growing tremendously with improvements in the quality of life, and healing of skin wounds has also been of huge importance in terms of bedsore treatment due to the acceleration of aging. Therefore, several studies on anti-aging and wound healing are under way.

Recently, non-thermal atmospheric plasma has shown beneficial effects on the healing of skin wounds; therefore, it has been considered as a potential tool for skin treatment. According to 
Heinlin's report, repeated plasma treatment for two minutes a day on the site of venous ulcers resulted in excellent wound healing. A previous study showed that repeated plasma treatment performed eleven times resulted in no bacteria in the wound area [15]. In our previous study, treatment of skin cells with low-temperature microwave plasma increased the expression of collagen fibers, fibronectin, and vascular endothelial growth factor (VEGF) genes; no thermal damage to the cells, due to the plasma or change in the $\mathrm{pH}$ of the medium, was observed [16]. These studies clearly suggest that plasma can be a great tool for anti-aging and healing of skin wounds. However, there have been many studies on the phenomenon occurring in the skin caused by plasma, but the mechanism causing such phenomenon has been poorly reported. In our previous studies, we have shown that in the process of wound healing, non-thermal plasma (NTP) inhibited E-cadherin of keratinocytes in the epidermis, thereby $\beta$-catenin binds to E-cadherin to migrate to the nucleus because of the weak bond and acts as a transcription factor for cell division [17].

In this study, we focused on the expression of several growth factors in epidermal tissue, since along with the contribution of E-cadherin and $\beta$-catenin, the involvement of cell growth factors seems to be essential for NTP-mediated biological changes in dermal tissue. To this end, HRM2 hairless mice were subjected to repeated treatment with NTP. After the treatment, NTP-mediated changes in skin tissue, along with the epidermis and dermis, was monitored. Furthermore, NTP-mediated changes in the expression of growth factors, including transcription growth factor (TGF)- $\alpha$, TGF- $\beta$, VEGF, granulocyte-macrophage colony-stimulating factor (GM-CSF), and epidermal growth factor (EGF), all of which are well known in wound healing and dermal tissue remodeling, were monitored. Taken together, this study suggests that NTP-mediated secretion of several growth factors in the epidermis can be a cause of changes in deep skin.

\section{Methods}

\section{NTP device}

The NTP device used in this study consisted of two electrodes and an alumina tube, which is a coaxial dielectric barrier discharge configuration. A stainless-steel rod with $3 \mathrm{~mm}$ inner diameter was used as the inner electrode and copper tape with a width of $10 \mathrm{~mm}$ was used as the outer electrode. An alumina $\left(\mathrm{Al}_{2} \mathrm{O}_{3}\right)$ tube $(4 \mathrm{~mm}$ inner diameter and $6 \mathrm{~mm}$ outer diameter) served as a dielectric, which prevented the transition of glow to arc discharges. The argon working gas was delivered at a flow rate of 2 standard liters per minute using a mass flow meter. The directions of the gas flow and the electric field are perpendicular to each other. A sinusoidal high voltage of $3 \mathrm{kV}$ with a frequency of $15 \mathrm{kHz}$ was applied to the inner electrode and the outer copper electrode was grounded (Figure 1). Non thermal plasma generated in this device does not extend from the nozzle like a plasma jet, but generated between the inner electrode and the alumina tube with discharge volume of $7.85 \mathrm{~mm}^{3}$.

\section{Mouse experiments}

Five-week-old male HRM2 melanin-possessing hairless mice were obtained from Central Laboratory Animal Inc. (Seoul, Korea). All experimental protocols in this study were approved by the Animal Ethics Committee, Pusan National University (PNU 017-1446). Mice ( $\mathrm{n}=5$ per group) were subjected to repeated treatments with gas only (GO) and NTP for a total of six times for $5 \mathrm{~min}$ each. After the final treatment at two weeks, the mice were sacrificed, and their skin tissues were collected for histology.

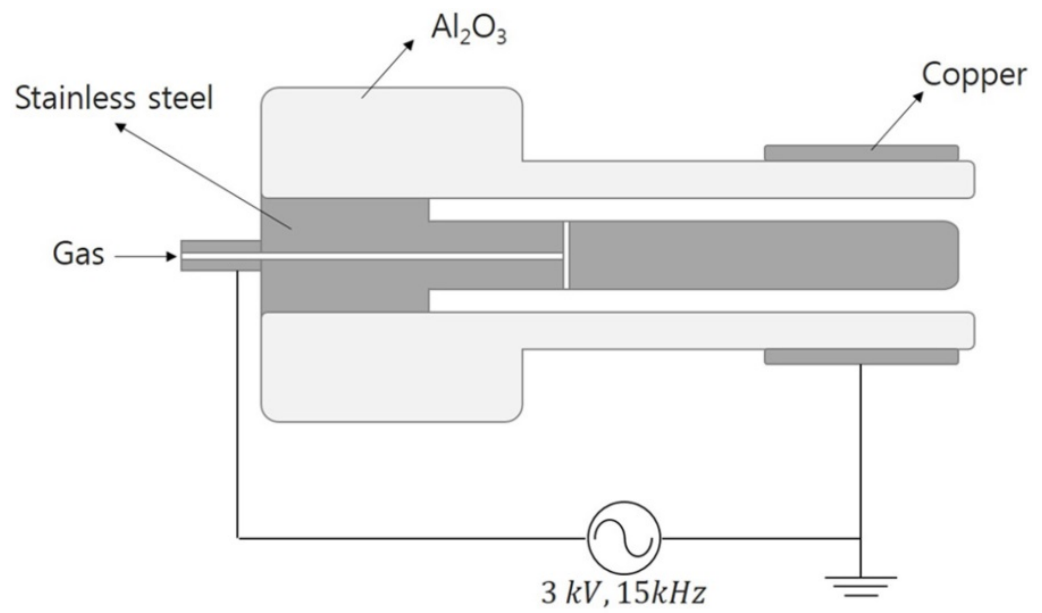

Figure 1. The structure of the non-thermal plasma (NTP) device. A schematic of the NTP device and operating process in this experiment. 


\section{Hematoxylin and eosin staining}

Mouse skin sections were stained with hematoxylin and eosin (H\&E). Tissue sections with a thickness of $5 \mu \mathrm{m}$ were fixed with $10 \%$ formalin, embedded with paraffin, cut on salinized glass slides, deparaffinized three times with xylene, and rehydrated through graded ethanol. After deparaffinization, rehydration, and rinsing with distilled water, the sections were stained with Harris hematoxylin for $3 \mathrm{~min}$, and then stained in an aqueous solution of eosin for $30 \mathrm{~s}$. The sections were dehydrated in ethanol and cleared in xylene. The samples were imaged using an Axio Scan Z1 slide scanner (Goettingen, Germany).

\section{DAPI staining}

Mouse sections were stained with DAPI (4',6-diamidino-2-phenylindole) for nucleic acids and mounted with ProLong ${ }^{\mathrm{TM}}$ Gold Antifade Mountant contained in the DAPI. Fluorescent images were acquired and analyzed using a Zeiss LSM 700 laser scanning confocal microscope (Goettingen, Germany).

\section{Masson's trichrome staining}

The slides were treated overnight with Bouin's solution at $25^{\circ} \mathrm{C}$, and then rinsed under tap water for $10 \mathrm{~min}$ to remove the yellow color. To stain the nuclei, slices were stained with Weigert iron hematoxylin for $10 \mathrm{~min}$. Then the slides were stained in Biebrich scarlet acid fuchsin solution for $2 \mathrm{~min}$, and differentiated in phosphomolybdic-phosphotungstic acid solution. Slides were disposed with aniline blue for $2 \mathrm{~min}$ and differentiated in 1\% acetic acid. Subsequently, the slides were dehydrated and cleared. The samples were imaged using an Axio Scan Z1 slide scanner (Goettingen, Germany).

\section{Immunohistochemical analysis}

Mouse skin from NTP-treated groups was prepared for immunohistochemical analysis of the expression of TGF- $\alpha$, TGF- $\beta$, VEGF, GM-CSF, and EGF. Tissue sections ( $5 \mu \mathrm{m}$ in thickness) were fixed with $10 \%$ formalin, embedded with paraffin, cut on slides, deparaffinized three times with xylene, and rehydrated through graded alcohol. To diminish non-specific staining, each section was treated with $0.3 \%$ hydrogen peroxide for $10 \mathrm{~min}$ and protein blocking solution (Abcam, Cambridge, MA) for 10 $\mathrm{min}$. The sections were incubated with the following primary antibodies: rabbit polyclonal anti-TGF- $\alpha$ (1:400), TGF- $\beta$ (1:250), VEGF (1:100), GM-CSF (1:100), and EGF (1:100) overnight at $4^{\circ} \mathrm{C}$ in Antibody Diluent (Dako, Glostrup, Denmark), and each section was then treated with biotinylated secondary antibody (1:100) (Dako, Glostrup, Denmark). The sections were incubated with avidin-biotin horseradish peroxidase complex (ABC) (Vector Laboratories, Burlingame, CA) for $30 \mathrm{~min}$. The peroxidase binding sites were detected by staining with 3,39-diaminobenzidine tetrahydrochloride (DAB) (Dako, Glostrup, Denmark). The samples were then counterstained with Mayer's hematoxylin (Dako, Glostrup, Denmark) imaged using an Axio Scan Z1 slide scanner (Goettingen, Germany).

\section{Statistical analysis}

One-way ANOVA and post-hoc Tukey's test were used to compare treatment effects for experiments. The results are presented as mean \pm standard error (SE). $p<0.05$ was considered to be statistically significant.

\section{Results and discussion}

\section{Histology of mouse skin after NTP treatment}

The mouse tissue sections were stained with H\&E and Masson's trichrome staining solutions to examine the change in the general morphology of mouse skin and dermal collagen fiber. In both histological analyses, we found that the epidermal layer of mouse skin treated with NTP was much thicker than that without NTP or with gas only (Figure 2A). The increment in epidermal thickness was a result of the proliferation of keratinocytes in the epidermal layer. Hoechst staining showed that the nuclei of cells were actively dividing (Figure 2B). These results demonstrated that NTP could induce skin cell proliferation, as E-cadherin was inhibited by plasma treatment so that $\beta$-catenin was released to the cytosol and played a role as a transcriptional factor. As shown in figure $2 \mathrm{C}$, quantitative measurement indicated that the epidermal thickness of skin treated with NTP was twice that of non-treated and gas-treated groups $(p<0.001)$. These results suggested that NTP effectively caused the proliferation of the epidermal layer, so that it would be useful not only for wound healing, but also for maintaining healthy skin by protecting skin from aging.

Collagen is a structural component of the dermis and a very important factor in anti-aging and skin regeneration. To further examine the histological appearance of collagen fibers in mouse skin tissue, we performed Masson's trichrome staining. The blue color indicated the expression of collagen fibers. The density of dermal collagen fibers in the non-treated group was lower than that in the NTP-treated group. Furthermore, the deposition of the collagen fibers was compact and thicker in the NTP-treated group (Figure 3). Masson's trichrome staining showed that NTP promoted the production of collagen in the dermal layer. Fibroblasts in the dermal layer produce various 
A
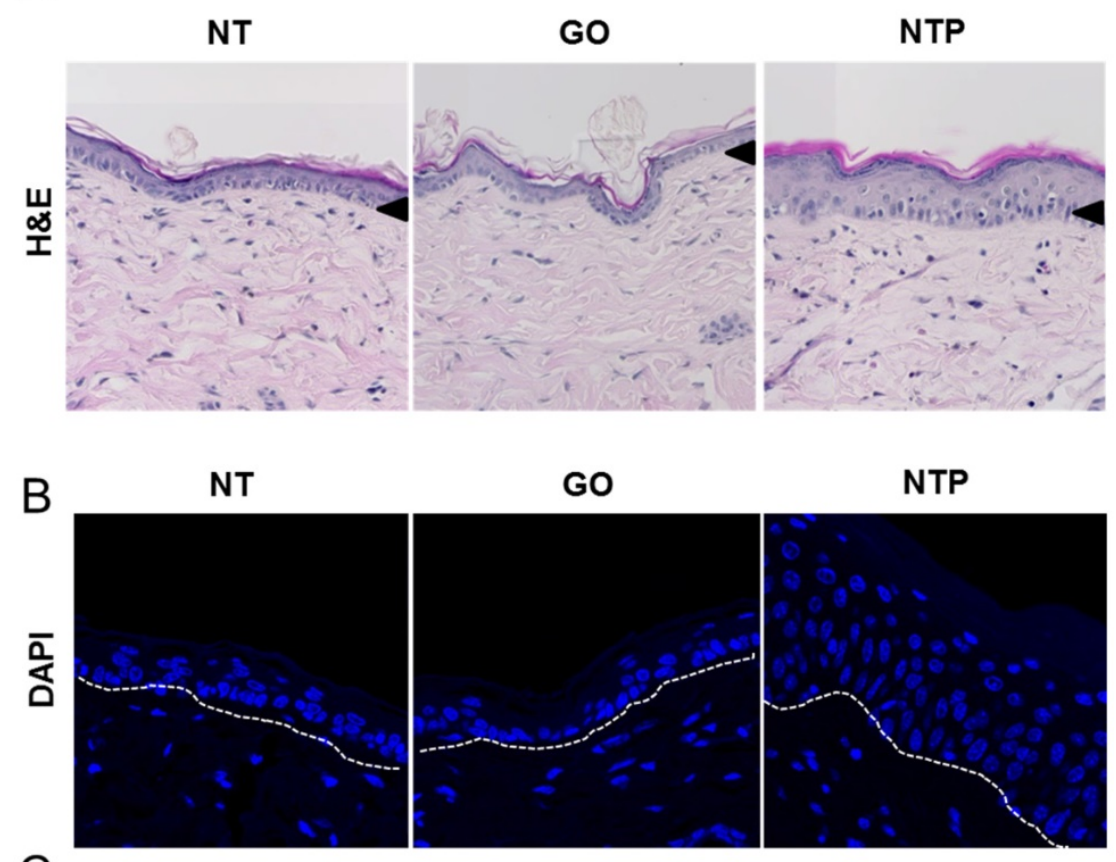

C

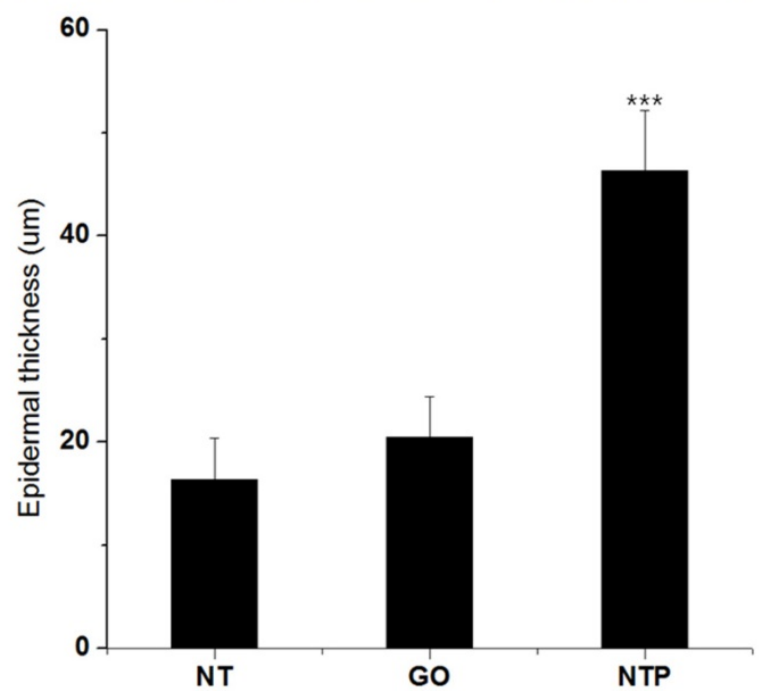

Figure 2. The effect of NTP on mouse skin at day 14. A. After sacrificing the mouse, skin sections obtained from the non-treated group (NT), gas-treated group (GO), and NTP-treated group (NTP) were stained with hematoxylin and eosin (H\&E), 100x. B. After sacrificing the mouse, skin sections obtained from the non-treated group (NT), gas-treated group (GO), and NTP-treated group (NTP) were stained with DAPI, 100×. C. Quantitative analysis of epidermal thickness in the non-treated group (NT), gas-treated group (GO), and NTP-treated group (NTP). ${ }^{* * *} p<0.001$ (ANOVA).

extracellular matrix proteins such as collagen fibers, elastin fibers, and fibronectin [18-19]. In the present study, the increase in collagen fibers in the dermal layer was likely caused by the activation of fibroblasts after NTP treatment. In turn, they synthesized and produced a large amount of collagen in the dermis. Since the skin barrier function is mainly mediated by the epidermal tissue, NTP treatment cannot affect fibroblasts directly. Therefore, we assumed that growth factors were produced from the epidermis after NTP treatment, and they then stimulated the fibroblasts to produce and secrete several types of extracellular matrix components. Thus, we investigated the expression of various growth factors in mouse skin after NTP treatment by means of immunohistochemistry.

\section{Immunohistochemical staining of mouse skin after NTP treatment}

Immunohistochemical analysis was performed to monitor the changes in growth factors in the epidermis and dermis after NTP treatment in mouse skin. A growth factor is a substance that stimulates cellular growth, proliferation, and differentiation under controlled conditions [20]. It is secreted by all cell types and known to play a significant role in anti-aging and tissue regeneration [21]. A number of growth factors are secreted from the epidermis and dermis, including TGF- $\alpha$, TGF- $\beta$, VEGF, GM-CSF, fibroblast growth factor (FGF)-2, platelet-derived growth factor, keratinocyte growth factor, and EGF [22-24]. In the present study, after treatment with NTP for two weeks, the expression of TGF- $\alpha$, TGF- $\beta$, VEGF, GM-CSF, and EGF were increased in mouse skin (Figure 4). TGF-a is a single-chain polypeptide that is a ligand for the EGF receptor related to EGF [25]. The general function of TGF-a is to activate a signaling pathway for cell restitution, proliferation, differentiation, and development [26]. After NTP treatment, it was strongly expressed over the epidermal layer, especially filling the cytosol of keratinocytes. Considering that the thickness of the dermal layer was increased by NTP treatment, keratinocytes were stimulated by NTP and actively produced TGF-a, which supposedly caused neighboring cells to proliferate.

TGF- $\beta$ stimulates fibroblasts and keratinocytes to induce cell migration, wound healing, and tissue repair [27]. In our previous study, wounded skin treated with NTP healed much faster than that without NTP treatment [17]. In the healing of skin wounds, re-epithelialization is very important as it is essential for cell migration. Keratinocytes, Langerhans cells, and Merkel cells exist in the epidermis, and 

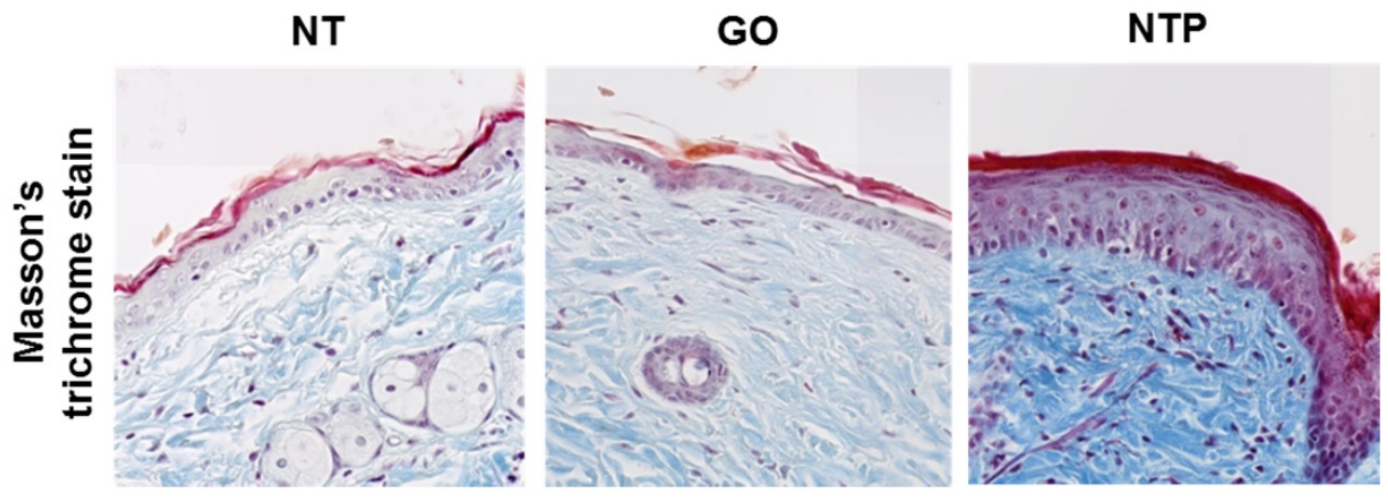

Figure 3. The expression of collagen on mouse skin after NTP treatment at day 14. After sacrificing the mouse, skin sections obtained from the non-treated group (NT), gas-treated group (GO), and NTP-treated group (NTP) were stained with Masson's trichrome stain (MTS), 100x.

among them, keratinocytes are the major component [28]. The results of the present study showed that TGF- $\beta$ was expressed in the nuclei of several types of cells in the epidermis. It was unclear which cells expressed TGF- $\beta$, but it was distributed in the nuclei of cells. Furthermore, TGF- $\beta$ was also expressed in cells of the dermis. As shown in figure 3, collagen density was increased after NTP treatment. Collagen fibers in the dermis are synthesized by fibroblasts, thus the increase in TGF- $\beta$ by NTP could activate fibroblasts to produce collagen fibers.

VEGF, also known as vascular permeability factor, is a disulfide-linked dimeric glycoprotein of approximately $40 \mathrm{kDa}$ that induces angiogenesis, endothelial cell proliferation, and activation of monocytes/macrophages [29-31]. In the present study, VEGF was expressed over the epidermal layer, but not in the basal layer, which is the lowermost layer of keratinocytes. This result is in agreement with the in-vitro data in our previous study, which showed that NTP treatment on HaCaT keratinocytes and human dermal fibroblasts increased VEGF mRNA expression. Synthesized VEGF most likely stimulates new blood vessel formation in NTP-treated areas in the dermis and supplies nutrients to fibroblasts. GM-CSF is a 23-kDa glycoprotein that plays a significant role as an immune-modulator, activating macrophages and granulocytes. Moreover, GM-CSF promotes angiogenesis, keratinocyte growth, epidermis regeneration, and wound healing [32, 33]. In the present study, GM-CSF was strongly expressed in the epidermal layer, including the basal layer, unlike the case of VEGF. It seems that the expression of GM-CSF after NTP treatment was strongly related to wound healing by NTP. EGF is a small protein with a molecular mass of $6 \mathrm{kDa}$, which increases the renewal rate of aging cells and accelerates wound healing in skin [34]. In addition, it stimulates the proliferation of epidermal cells and differentiation of skin appendages [35]. In particular, EGF has been considered to be important in esthetics, and it has been widely used for skin rejuvenation. In the present study, EGF was expressed over the epidermal layer after NTP treatment but its expression was not observed in the basal layer. The expressed EGF by NTP could lead to cell proliferation in the epidermis.

Above all, NTP treatment on skin not only caused cell proliferation in the epidermis and the increase in collagen fibers in the dermis, but also promoted active expression of various growth factors. These results are very promising in skin rejuvenation and wound healing. Therefore, enhanced expression of growth factors by treatment of NTP is thought to be an important mechanism in wound healing. As this study was focused on the expression of growth factors in skin tissues by NTP, the observation was performed after two weeks of NTP treatment. Thus, in the next experiment, not only long-term examination of normal tissues will be performed, but also various wound tissues will be observed.

\section{Conclusions}

This study provides evidence for the histological anti-aging effect and safety of the treatment of NTP in mouse skin tissue. This treatment does not induce thermal damage to mouse skin tissue. In addition, the epidermal layer thickness and density of collagen in the dermis were increased after NTP treatment. Furthermore, we observed significant increases in the levels of growth factors such as TGF-a, TGF- $\beta$, VEGF, GM-CSF, and EGF after the treatment. Taken together, since NTP not only directly activates the proliferation of epidermal cells but also accelerates dermal remodeling by stimulating the secretion of several types of growth factors, this study suggests that NTP can be an innovative tool for wound healing and anti-aging of the skin. 


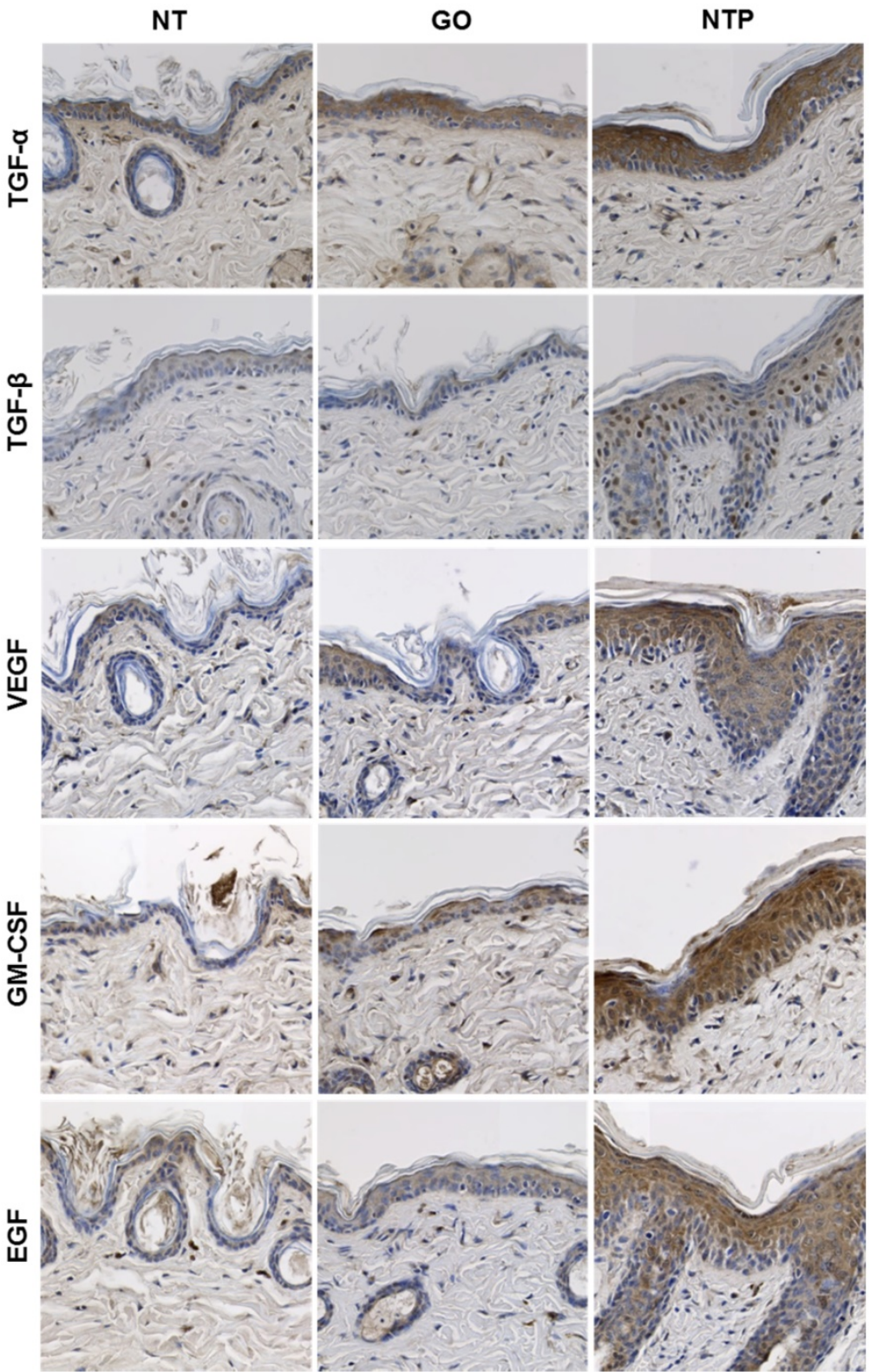

Figure 4. The expression of growth factors on mouse skin after NTP treatment at day 14. After sacrificing the mouse, skin sections obtained from the non-treated group (NT), gas-treated group (GO), and NTP-treated group (NTP) were subjected to immunohistochemistry (IHC) for TGF- $\alpha$, TGF- $\beta$, VEGF, GM-CSF, and EGF, 100×.

\section{Acknowledgments}

This research was supported by the Bio \& Medical Technology Development Program of the National Research Foundation (NRF) \& funded by the Korean government (2016M3A9C6918283)

\section{Abbreviations}

NTP: non-thermal plasma; VEGF: vascular endothelial growth factor; (TGF)-a: transcription growth factor alpha; (TGF)- $\beta$ : transcription growth factor beta; GM-CSF: granulocyte-macrophage colony-stimulating factor; EGF: epidermal growth factor; H\&E: hematoxylin and eosin; DAPI: 4',6-diamidino-2-phenylindole; ABC: avidin-biotin horseradish peroxidase complex; DAB: 3,39-diaminobenzidine tetrahydrochloride.

\section{Competing Interests}

The authors have declared that no competing interest exists.

\section{References}

1. RR Wickett, MO Visscher. Structure and function of the epidermal barrier. American journal of infection control 2006.

2. Boer M, Duchnik E, Maleszka $R$, et al. Structural and biophysical characteristics of human skin in maintaining proper epidermal barrier function. Postepy Dermatol Alergol 2016; 33: 1-5.

3. Kleesz P, Darlenski R, Fluhr JW. Full-body skin mapping for six biophysical parameters: baseline values at 16 anatomical sites in 125 human subjects. Skin Pharmacol Physiol 2012; 25: 25-33.

4. Watt FM. The epidermal keratinocyte. Bioessays 1988;8: 163-167. 
5. Régnier M, Patwardhan A, Scheynius A, et al. Reconstructed human epidermis composed of keratinocytes, melanocytes and Langerhans cells. Med Biol Eng Comput 1998;36: 821-824.

6. Brohem CA, Cardeal LB, Tiago M, et al. Artificial skin in perspective: concepts and applications. Pigment Cell Melanoma Res 2011;24: 35-50.

7. Lee DY, Yang JM, Park KH. A dermal equivalent developed from fibroblast culture alone: effect of EGF and insulin. Wound Repair Regen 2007;15: 936-939.

8. Gerber PA, Buhren BA, Schrumpf $\mathrm{H}$, et al. Mechanisms of skin aging induced by EGFR inhibitors. Support Care Cancer 2016;24: 4241-4248.

9. Chen J, Li Y, Zhu Q, et al. Anti-skin-aging effect of epigallocatechin gallate by regulating epidermal growth factor receptor pathway on aging mouse model induced by d-Galactose. Mech Ageing Dev 2017; 164: 1-7.

10. Aldag C, Nogueira Teixeira D, Leventhal PS. Skin rejuvenation using cosmetic products containing growth factors, cytokines, and matrikines: a review of the literature. Clin Cosmet Investig Dermatol 2016; 9: 411-419.

11. Lee $\mathrm{DH}$, Oh IY, Koo KT, et al. Improvement in skin wrinkles using a preparation containing human growth factors and hyaluronic acid serum. J Cosmet Laser Ther 2015;17: 20-23.

12. Somchaichana J, Bunaprasert T, Patumraj S. Acanthus ebracteatus Vahl. ethanol extract enhancement of the efficacy of the collagen scaffold in wound closure: a study in a full-thickness-wound mouse model. J Biomed Biotechnol 2012; 754527.

13. Xue M, Jackson CJ. Extracellular Matrix Reorganization During Wound Healing and Its Impact on Abnormal Scarring. Adv Wound Care (New Rochelle) 2015; 4: 119-136.

14. Saito T, Izumi K, Shiomi A, et al. Zoledronic acid impairs re-epithelialization through down-regulation of integrin av $\beta 6$ and transforming growth factor beta signalling in a three-dimensional in vitro wound healing model. Int J Oral Maxillofac Surg 2014; 43: 373-380.

15. Heinlin J, Morfill G, Landthaler $\mathrm{M}$, et al Plasma medicine: possible applications in dermatology. J Dtsch. Dermatol Ges 2010; 8: 968-976.

16. Choi JH, Lee HW, Lee JK, et al. Low-temperature atmospheric plasma increases the expression of anti-aging genes of skin cells without causing cellular damages. Arch. Dermatol Res 2013; 305: 133-140.

17. Choi JH, Song YS, Song K, et al. Skin renewal activity of non-thermal plasma through the activation of $\beta$-catenin in keratinocytes. Sci Rep 2017;7: 6146.

18. Roh SS, Lee MH, Hwang YL, et al. Stimulation of the extracellular matrix production in dermal fibroblasts by velvet antler extract. Ann Dermatol 2010;22: 173-179.

19. Jeon YK, Jang YH, Yoo DR, et al. Mesenchymal stem cells' interaction with skin: wound-healing effect on fibroblast cells and skin tissue. Wound Repair Regen 2010;18: 655-661.

20. Park JW, Hwang SR, Yoon IS. Advanced Growth Factor Delivery Systems in Wound Management and Skin Regeneration. Molecules 2017;27: E1259.

21. Gibbs S, Silva Pinto AN, Murli S, et al. Epidermal growth factor and keratinocyte growth factor differentially regulate epidermal migration, growth, and differentiation. Wound Repair Regen 2000;8: 192-203.

22. Aldag C, Nogueira Teixeira D, Leventhal PS. Skin rejuvenation using cosmetic products containing growth factors, cytokines, and matrikines: a review of the iterature. Clin Cosmet Investig Dermatol 2016; 9: 411-419

23. Safari M, Ghahari L, Zoroufchi MD. Effects of epidermal growth factor, platelet derived growth factor and growth hormone on cultured rat keratinocytes cells in vitro. Pak J Biol Sci 2014;17: 931-936.

24. Andreadis ST, Hamoen KE, Yarmush ML, et al. Keratinocyte growth factor induces hyperproliferation and delays differentiation in a skin equivalent model system. FASEB J 2001; 15: 898-906.

25. Borlinghaus $\mathrm{P}$, Wieser $\mathrm{S}$, Lamerz R. Epidermal growth factor, transforming growth factor-alpha, and epidermal growth factor receptor content in normal and carcinomatous gastric and colonic tissue. Clin Investig 1993;71: 903-907.

26. Vassar R, Fuchs E. Transgenic mice provide new insights into the role of TGF-alpha during epidermal development and differentiation. Genes Dev 1991;5: 714-727.

27. Amjad SB, Carachi R, Edward M. Keratinocyte regulation of TGF-beta and connective tissue growth factor expression: a role in suppression of scar tissue formation. Wound Repair Regen 2007; 15: 748-755.

28. Zhang C, Tan CK, McFarlane C, et al. Myostatin-null mice exhibit delayed skin wound healing through the blockade of transforming growth factor- $\beta$ signaling by decorin. Am J Physiol Cell Physiol 2012;302: C1213-1225.

29. Cao $Y$, Linden $P$, Farnebo J, et al. Vascular endothelial growth factor $C$ induces angiogenesis in vivo. Proc Natl Acad Sci USA 1998;95: 14389-14394.

30. Cébe-Suarez S, Zehnder-Fjällman A, Ballmer-Hofer K. The role of VEGF receptors in angiogenesis; complex partnerships. Cell Mol Life Sci 2006; 63: 601-615

31. Inoue $\mathrm{M}$, Itoh $\mathrm{H}$, Tanaka $\mathrm{T}$, et al. Oxidized $\mathrm{LDL}$ regulates vascular endothelial growth factor expression in human macrophages and endothelial cells through activation of peroxisome proliferator-activated receptor-gamma. Arterioscler Thromb Vasc Biol 2001;21: 560-566.

32. Braunstein $S$, Kaplan $G$, Gottlieb AB, et al. GM-CSF activates regenerative epidermal growth and stimulates keratinocyte proliferation in human skin in vivo. J Invest Dermatol 1994;103: 601-604.

33. Zhao J, Chen L, Shu B, et al. Granulocyte/macrophage colony-stimulating factor influences angiogenesis by regulating the coordinated expression of VEGF and the Ang/Tie system. PLoS. One. 2014;9: e92691.
34. J.D. Zieske, H. Takahashi, A.E. Hutcheona, et al. Activation of epidermal growth factor receptor during corneal epithelial migration. Invest Ophthalmol Vis Sci 2000; 41: 1346-1355.

35. Tran QT, Kennedy LH, Leon Carrion S, et al. EGFR regulation of epidermal barrier function. Physiol Genomics 2012;44: 455-469. 